\title{
Reflexiones sobre el proceso investigativo con la comunidad indígena U'WA
}

Por: Christian Camilo Caicedo Moreno

Como docente, la experiencia investigativa hace parte de los distintos escenarios académicos en los que me desenvuelvo, sobre todo el trabajo con comunidades, del que, al ser de los procesos más ricos y robustos, siempre quise formar parte. Durante este proceso de inmersión investigativo, junto a la docente del programa de Diseño de Modas Angela Dotor, se tuvo como objetivo principal rescatar costumbres y tradiciones propias de nuestra cultura. De allí nació el proyecto de investigación y el interés profundo por entender qué tradiciones, técnicas de tejeduría, fibras textiles y componentes de patrimonio cultural, hacen parte y constituyen la identidad de la comunidad indígena U’WA, ubicada geográficamente en la sierra nevada del Cocuy y Cubará.

Este proyecto abogó por la reconstrucción y mantenimiento del patrimonio, identidad y cultura de la comunidad indígena U`wa, partiendo de una mirada externa y de la curiosidad académica relacionada con el diseño de modas, a través de un acercamiento etnográfico que rebelará aspectos de esta cultura enmarcados en el manejo de sus tejidos ancestrales. Desde esta perspectiva se hará una investigación documentada 
que visibilice y promueva el importante trabajo de esta comunidad, situada en su propio contexto particular, para fomentar el interés investigativo por sus costumbres, tanto institucional como externo, que tiene a la vez la clara intención de generar procesos de diseño que re signifiquen tradiciones propias de nuestro país. A modo de diario, este trabajo hizo parte de un ejercicio de reconstrucción de memoria relacionado con comunidades que para muchos son, en gran medida, desconocidas.

En el trabajo de campo desarrollado y en nuestra primera visita al sitio, los inconvenientes y problemas no tardaron en presentarse. Parte del ejercicio investigativo de recolección e interacción se vio abruptamente interrumpido por las acciones de diferentes instituciones que se encontraban allí, las cuales, de acuerdo con la comunidad, tenían como fin recolectar información para documentar y sacar provecho de estas comunidades, sin ser participes ni brindar un valor agregado a los indígenas. Esta población siente que las personas ajenas a su comunidad solo buscan "exotizarlas", pasar por encima de sus creencias y tradiciones, y por ende, sienten que poco a poco desangran la identidad U'wa, fomentando la desaparición de la misma. Antropólogas como Silvia Parra y Helena Pradilla, han hecho estudios en la comunidad relacionados con los estudios de género, la educación y análisis de artefactos o productos que han generado datos valiosos e importantes, pero que en su núcleo denotan la falta de estudio y profundización, entorno a fibras textiles, tejidos y tintorería de la comunidad U 'wa.

Desde hace algunos años las comunidades ancestrales y pueblos originarios del país vienen atravesando un proceso de aculturación propio del crecimiento de la población y la mezcla entre comunidades indígenas con culturas urbanas. Este proceso de hibridación responde a lógicas de un mundo globalizado que imposibilita que las comunidades se mantengan ajenas o separadas del mundo y el entorno, elemento que, desde la perspectiva $U^{\prime}$ wa, pone en peligro el núcleo de su identidad ancestral. 
El recelo de esta comunidad hacia los "extranjeros" es un factor que podría desvincular a la academia de diseño de indumentaria, del acceso a la historia, saberes y aprendizajes U'wa y privarla del acceso a posibles lugares de creación de conocimiento como apoyo a la reconstrucción de memoria de nuestro país.

La comunidad indígena U'wa, ubicada en el Nororiente del país, es poseedora de conocimientos en cuanto a tejeduría, tintorería, tratamiento, uso de fibras naturales e indumentaria, que en este proyecto son recolectados e inventariados dada la importancia de dar una mirada al pasado, en búsqueda de procesos sostenibles amigables con el entorno. Para buscar herramientas que construyan un futuro de la moda en Colombia, hay que trabajar un factor importante: la identidad del país y su riqueza cultural. Es imperativo actualmente identificar y potencializar aquello que nos hace únicos y que nos permita sobresalir a nivel global como precursores y cuidadores de lo que nos hace verdaderamente colombianos; por esta razón, es vital remontarnos al origen y a nuestras tradiciones ancestrales.

A partir del trabajo de campo desarrollado y a pesar del temor de la comunidad, fue posible recopilar información acerca del proceso de extracción y preparación de fibras naturales que son extraídas de tallos u hojas de las plantas. Este proceso consiste en una sucesión de procesos muy parecidos. En primer lugar, la corteza u hoja de las plantas pasa por un proceso de recolección y remojo, luego son golpeadas para suavizarlas, pasan por una fase de secado y desmenuzado, y finalmente, la materia es torcida hasta producir hilos de fibra.

Son dos los tipos de fibra más trabajados por la comunidad indígena U’wa en procesos de tejeduría.

Fibra de Tchivara o cabuya de fique, cuenta con características de resistencia y economía, además de servir como alimento y abono.

Fibra de Moriche: palma de gran tamaño con frutos escamados, utilizada en la elaboración de chinchorros. También es capaz de generar alimento y madera, su comercialización es escasa. 
"Es vital remontarnos al origen y a nuestras tradiciones ancestrales." 
El acercamiento a esta comunidad, brindó no solo los resultados esperados frente a la investigación de sus fibras, sino que dio importancia a la necesidad de generar debates frente a la labor que realiza la academia del diseño de indumentaria dentro del trabajo etnográfico con comunidades, partiendo de un principio de conservación y responsabilidad frente a las cosmovisiones de estas culturas, así como la importancia de generar espacios de diálogo permanente en búsqueda de acuerdos y beneficios comunes. Así, podrá darse paso a investigaciones más profundas que vinculen a estudiantes de distintas áreas, en la construcción de un sentido propio del trabajo comunitario en la importante labor de buscar una moda Latinoamericana y sostenible.

Parte de los mayores aprendizajes obtenidos durante esta investigación, tuvieron que ver con la certeza de que efectivamente es posible incorporar a la población estudiantil dentro de procesos de investigación de los saberes ancestrales de comunidades indígenas, sin embargo, se dificulta gravemente la intervención en la decisión estética para el desarrollo de producto de la comunidad, aspecto de principal interés de estudiantes y docentes. ¿Pero quién debe decidir sobre lo que las comunidades necesitan o buscan? Debemos comprender que las comunidades tienen una visión propia del diseño y que la idea de llegar a "modernizarlos" desemboca en una mala imagen de la academia y las instituciones. Debemos empezar por conocer a las comunidades como agentes externos y poco a poco asegurar su confianza con el cuidado de no irrumpir en sus procesos de identidad, sino con la intención de aprender, estudiar y socializar con culturas que podrían no existir en los próximos años. 


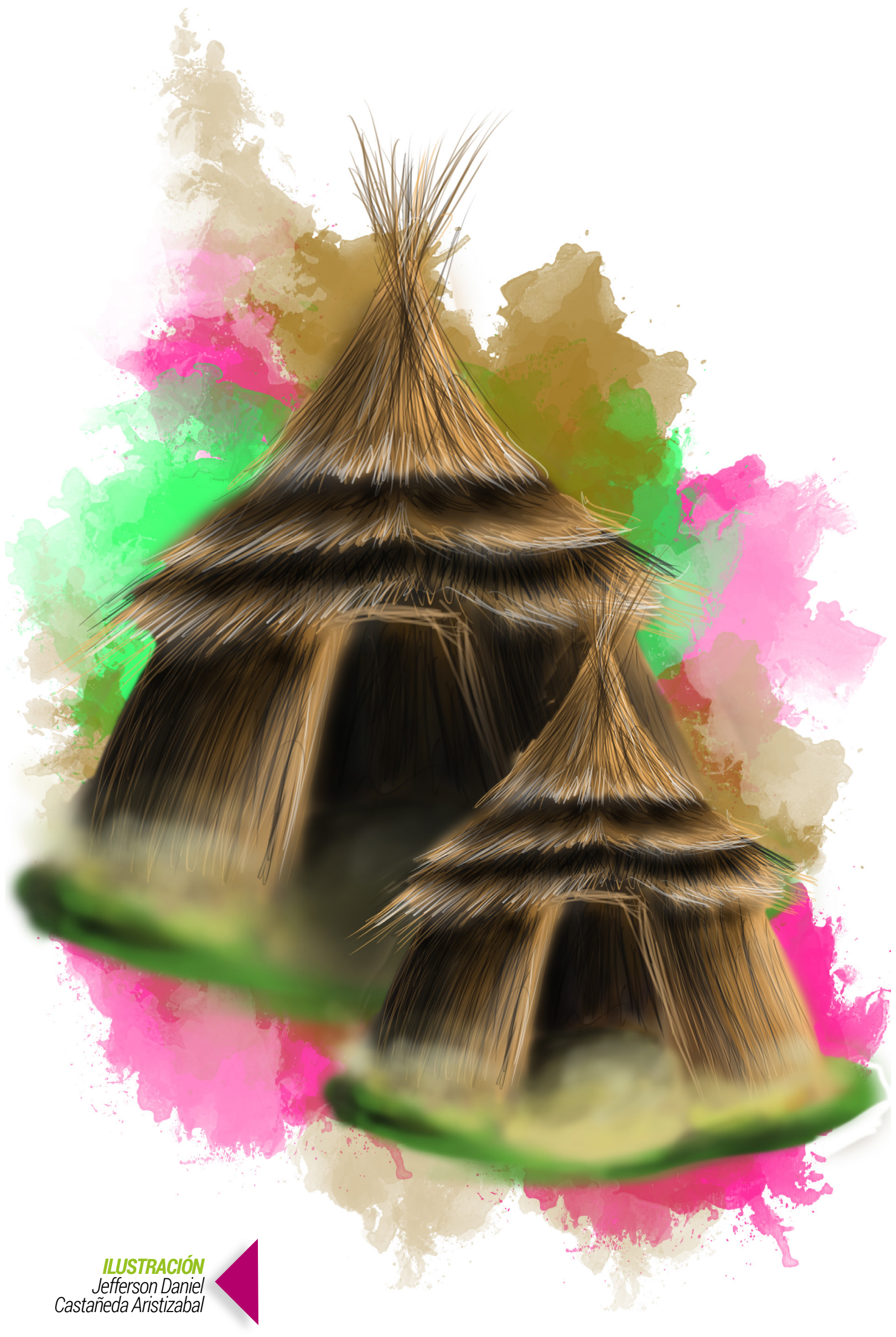

